\title{
Surface Bound States and Spontaneous Current in Cyclic $d$-Wave Superconductors
}

\author{
Masaki Ishikawa, ${ }^{1}$ Yasumasa Tsutsumi, ${ }^{2}$ Masanori Ichioka, ${ }^{1}$ and Kazushige MachidA ${ }^{1}$ \\ ${ }^{1}$ Department of Physics, Okayama University, Okayama 700-8530, Japan \\ ${ }^{2}$ Condensed Matter Theory Laboratory, RIKEN, Wako, Saitama 351-0198, Japan
}

\begin{abstract}
On the basis of Eilenberger theory, surface bound states and spontaneous current are studied in cyclic $d$-wave superconductors as a broken time-reversal symmetry of superconductivity in cubic lattice symmetry. We discuss how the spontaneous current and the electronic states depend on the orientation of the surface relative to the symmetry of superconductivity. The condition for topological Fermi arcs of zero-energy surface bound states to appear is identified in the complex pairing function of the cyclic $d$-wave.
\end{abstract}

KEYWORDS: surface state, spontaneous current, cyclic $d$-wave superconductivity, Eilenberger theory

Among unconventional superconductivity, the broken time reversal symmetry (BTRS) of superconductivity is an important topic of study, because it has exotic properties, such as zero-energy surface bound states and spontaneous magnetic field by spontaneous current. In most of the previous studies of the BTRS superconductor, the pairing symmetry was assumed to be a chiral $p$-wave $p_{x} \pm \mathrm{i} p_{y}$, considering the cases of the superfluid ${ }^{3} \mathrm{He}$ A-phase ${ }^{1-3}$ or $\mathrm{Sr}_{2} \mathrm{RuO}_{4} \cdot{ }^{4,5}$ The spontaneous magnetic field of a BTRS superconductor is detected by muon spin rotation ( $\mu \mathrm{SR}$ ) experiment in $\mathrm{Sr}_{2} \mathrm{RuO}_{4}{ }^{4}$ $\mathrm{PrOs}_{4} \mathrm{Sb}_{12},{ }^{6,7} \mathrm{LaNiC}_{2},{ }^{8} \mathrm{PrPt}_{4} \mathrm{Ge}_{12},{ }^{9}$ and $\mathrm{LaNiGa}_{2} .{ }^{10}$ In a $\mu \mathrm{SR}$ experiment, $\mathrm{PrOs}_{4} \mathrm{Sb}_{12}, \mathrm{PrPt}_{4} \mathrm{Ge}_{12}$, and $\mathrm{LaNiGa}_{2}$ show different types of relaxation curves from those of $\mathrm{Sr}_{2} \mathrm{RuO}_{4}$ and $\mathrm{LaNiC}_{2}$. Therefore, there may be some variety in the types of BTRS. Since the crystal lattice symmetry of $\mathrm{PrOs}_{4} \mathrm{Sb}_{12}$ is cubic $\mathrm{O}_{\mathrm{h}}$ (or more exactly, $\mathrm{T}_{\mathrm{h}}$ ), the pairing symmetry may be different from the chiral $p$-wave of $\mathrm{Sr}_{2} \mathrm{RuO}_{4}$. Therefore, it is important that we examine the possibility of a new type of pairing symmetry of BTRS other than the chiral $p$-wave, and study the properties of the new pairing function to identify the pairing symmetry.

In this study, we consider the BTRS of superconductivity in cubic lattice symmetry. From the classification table of possible pairing symmetries under spin-orbit coupling on the basis of point group theory, ${ }^{1-13}$ as a BTRS state keeping $\mathrm{O}_{\mathrm{h}}$ symmetric superconductivity, we find $k_{x}^{2}+\omega_{ \pm} k_{y}^{2}+\omega_{ \pm}^{2} k_{z}^{2}$ in spinsinglet pairing and $k_{x} \hat{x}+\omega_{ \pm} k_{y} \hat{y}+\omega_{ \pm}^{2} k_{z} \hat{z}$ in spin-triplet pairing with $\omega_{ \pm}=\mathrm{e}^{ \pm \mathrm{i} 2 \pi / 3}$. The former is cyclic $d$-wave pairing, and it is stable in weak coupling theory. The latter is nonunitary spin-triplet pairing, and it is not stable in weak coupling theory. Thus, the case of cyclic $d$-wave pairing is studied here. The possibility of cyclic $d$-wave superconductivity and its exotic properties have been discussed in the study of BoseEinstein condensation ${ }^{14-16}$ and fermionic superfluidity ${ }^{17}$ in cold atomic gases. There, the non-Abelian $\frac{1}{3}$-fractional vortex is also possible in an isotropic atomic gas system. Thus, the BTRS of cyclic $d$-wave pairing is one of the interesting pairing symmetries for the theoretical study of topological superconductivity.

Studies of the surface state are important to identify the pairing symmetry of unconventional superconductivity. For example, in a $d_{x^{2}-y^{2}}$-wave superconductor, the surface bound state depends on the surface orientation, and zero-energy sur- face bound states appear for the $(1,1,0)$ surface. ${ }^{18,19}$ The characteristic dispersion relation of the surface bound state reflects the pairing symmetry of the bulk superconductivity. In the $d_{x y}$-wave pairing and the $p_{x}$-wave pairing, we have flat dispersion of the surface bound states for the $(1,0,0)$ surface. ${ }^{19,20}$ In superfluid ${ }^{3} \mathrm{He}$, surface bound states show topological Fermi arcs for the A-phase and a Majorana cone for the B phase. ${ }^{2,3}$ Therefore, studies of the surface bound state are also necessary for the complex pairing function of cyclic $d$-wave superconductivity.

The purpose of this study is to clarify the properties of surface bound states in a cyclic $d$-wave superconductor, as another example of the BTRS state, on the basis of Eilenberger theory. Since the structure of surface bound states depends on the relative angle of the surface and the pairing symmetry, we study the surface-orientation dependence of spontaneous current and electronic states in the surface bound state. We also discuss the condition under which the zero-energy surface bound state appears in the cyclic $d$-wave superconductivity.

In our study, wave numbers are denoted as $\left(k_{a}, k_{b}, k_{c}\right)$ for crystal coordinates. Under an $\mathrm{O}_{\mathrm{h}}$ crystal field, we assume the pairing function to be the cyclic $d$-wave given by

$$
\begin{aligned}
& \phi_{ \pm}(\mathbf{k})=\sqrt{\frac{5}{2}}\left(k_{c}^{2}+\omega_{ \pm} k_{a}^{2}+\omega_{ \pm}^{2} k_{b}^{2}\right) \\
& =\frac{1}{\sqrt{2}}\left(\frac{\sqrt{5}}{2}\left(3 k_{c}^{2}-1\right) \pm \mathrm{i} \frac{\sqrt{15}}{2}\left(k_{a}^{2}-k_{b}^{2}\right)\right) .
\end{aligned}
$$

Therefore, $\phi_{-}(\mathbf{k})=\phi_{+}^{*}(\mathbf{k})$. In Eq. (1), $\mathbf{k}$ is mapped on the Fermi sphere and normalized as $k_{a}^{2}+k_{b}^{2}+k_{c}^{2}=1$. From Eq. (1), the cyclic $d$-wave is a combination of $d_{x^{2}-y^{2}}$-wave and $d_{3 z^{2}-1^{-}}$ wave components. The amplitude $\left|\phi_{ \pm}(\mathbf{k})\right|$ has $\mathrm{O}_{\mathrm{h}}$ symmetry with 8 point nodes in $(1,1,1)$ and equivalent directions. The phase of $\phi_{ \pm}(\mathbf{k})$ has $\mathrm{T}_{\mathrm{h}}$ symmetry. For $120^{\circ}$ rotation around the $(1,1,1)$-axis $\left(k_{a} \rightarrow k_{b}, k_{b} \rightarrow k_{c}, k_{c} \rightarrow k_{a}\right), \phi_{+} \rightarrow \omega^{2} \phi_{+}$. For $90^{\circ}$ rotation around the $(0,0,1)$-axis $\left(k_{a} \rightarrow k_{b}, k_{b} \rightarrow-k_{a}\right)$, $\phi_{+} \rightarrow \phi_{-}\left(\neq \phi_{+}\right)$. In the calculation of the surface state, we use the coordinates $\left(k_{x}, k_{y}, k_{z}\right)$ and $(x, y, z)$, where the $x$-axis is fixed to be perpendicular to the surface at $x=0 .\left(k_{x}, k_{y}, k_{z}\right)$ is obtained by rotational transformation from $\left(k_{a}, k_{b}, k_{c}\right)$. The $x$-direction is defined as $(\cos \theta \cos \phi, \cos \theta \sin \phi, \sin \theta)$ in the crystal coordinates. 
Surface states are calculated on the basis of Eilenberger theory, following the method used to study the surface states of superfluid ${ }^{3} \mathrm{He} .{ }^{2}$ Since we consider the case of spin-singlet pairing here, the transport-like Eilenberger equation is reduced to the $2 \times 2$ matrix form

$$
-\mathrm{iv} \cdot \nabla \hat{g}=\frac{1}{2}\left[\left(\begin{array}{cc}
\mathrm{i} \omega_{n} & -\Delta(\mathbf{k}, \mathbf{r}) \\
\Delta^{*}(\mathbf{k}, \mathbf{r}) & -\mathrm{i} \omega_{n}
\end{array}\right), \hat{g}\right],
$$

for quasi-classical Green's functions

$$
\hat{g}=-\mathrm{i} \pi\left(\begin{array}{cc}
g\left(\mathbf{k}, \mathbf{r}, \omega_{n}\right) & \mathrm{i} f\left(\mathbf{k}, \mathbf{r}, \omega_{n}\right) \\
-\mathrm{i} f^{\dagger}\left(\mathbf{k}, \mathbf{r}, \omega_{n}\right) & -g\left(\mathbf{k}, \mathbf{r}, \omega_{n}\right)
\end{array}\right)
$$

with the normalization condition $\hat{g}^{2}=-\pi^{2} \hat{1}$. $\omega_{n}$ is the Matsubara frequency. We consider the case where an external magnetic field is not applied. For simplicity, we neglect the contribution of vector potentials. The pair potential takes the general form

$$
\Delta(\mathbf{k}, \mathbf{r})=\Delta_{+}(\mathbf{r}) \phi_{+}(\mathbf{k})+\Delta_{-}(\mathbf{r}) \phi_{-}(\mathbf{k}) .
$$

We treat $\Delta_{+}$as the dominant component and $\Delta_{-}$as the induced component near the surface, so that $\Delta_{-}=0$ and $\Delta_{+}$ is a bulk value when $x \rightarrow \infty$. Since we assume a spherical Fermi surface, the normalized Fermi velocity is $\mathbf{v}=\mathbf{k}$. We assume that the surface condition is specular, and a quasiparticle with $\left(-k_{x}, k_{y}, k_{z}\right)$ is reflected to $\left(k_{x}, k_{y}, k_{z}\right)$ at $x=0$. We solve the Riccati equation derived from the Eilenberger equation [Eq. (2)], and obtain the quasi-classical Green's functions. Energy, temperature, and length are in units of $\pi k_{\mathrm{B}} T_{\mathrm{c}}$, $T_{\mathrm{c}}$, and $\xi_{0}=\hbar v_{\mathrm{F}} / 2 \pi k_{\mathrm{B}} T_{\mathrm{c}}$, respectively. The pair potential is calculated by the gap equation

$$
\Delta_{ \pm}(\mathbf{r})=\lambda_{0} 2 T \sum_{\omega_{n}>0}^{\omega_{\mathrm{c}}}\left\langle\phi_{ \pm}^{*}(\mathbf{k}) f\right\rangle_{\mathbf{k}},
$$

where $\langle\cdots\rangle_{\mathbf{k}}$ indicates the Fermi surface average. $\lambda_{0}=N_{0} g_{0}$ is the dimensionless pairing interaction defined by the cutoff energy $\omega_{\mathrm{c}}$ as $1 / \lambda_{0}=\ln T+2 T \sum_{\omega_{n}>0}^{\omega_{\mathrm{c}}} \omega_{n}^{-1}$. We carry out calculations using the cutoff $\omega_{\mathrm{c}}=40 k_{\mathrm{B}} T_{\mathrm{c}}$. The calculations of Eqs. (2) and (5) are iterated at $T=0.2 T_{\mathrm{c}}$ until self-consistent results are obtained.

The current density of spontaneous current is given as

$$
\mathbf{J}(\mathbf{r})=j_{0} 2 T \sum_{\omega_{n}>0}\langle\mathbf{v} \operatorname{Im} g\rangle_{\mathbf{k}},
$$

and produces the spontaneous magnetic field. $j_{0}=8 \pi \mathrm{e} N_{0} / c$, and $N_{0}$ is the density of states at the Fermi level. The total current of the surface bound states is given by the integral

$$
\mathbf{J}_{\text {total }}=\int_{0}^{\infty} \mathbf{J}(\mathbf{r}) \mathrm{d} x \text {. }
$$

The local density of states (LDOS) is obtained as

$N(E, \mathbf{r})=\langle N(E, \mathbf{k}, \mathbf{r})\rangle_{\mathbf{k}}=N_{0}\left\langle\operatorname{Reg}\left(\mathbf{k}, \mathbf{r}, \mathrm{i} \omega_{n} \rightarrow E+\mathrm{i} \eta\right)\right\rangle_{\mathbf{k}}$,

where we use the solution of the Eilenberger equation [Eq. (2)] for the real energy $E$ in the self-consistently obtained pair potential. We use a small smearing of $\eta=0.007 . N(E, \mathbf{k}, \mathbf{r})$ is the k-resolved LDOS.

First, in Figs. 1(a) and 1(b), we present the self-consistent solution of the pair potential and spontaneous current for some surface orientations. At the surface region, when the length is of the order of the coherence length, $\Delta_{+}$is sup-
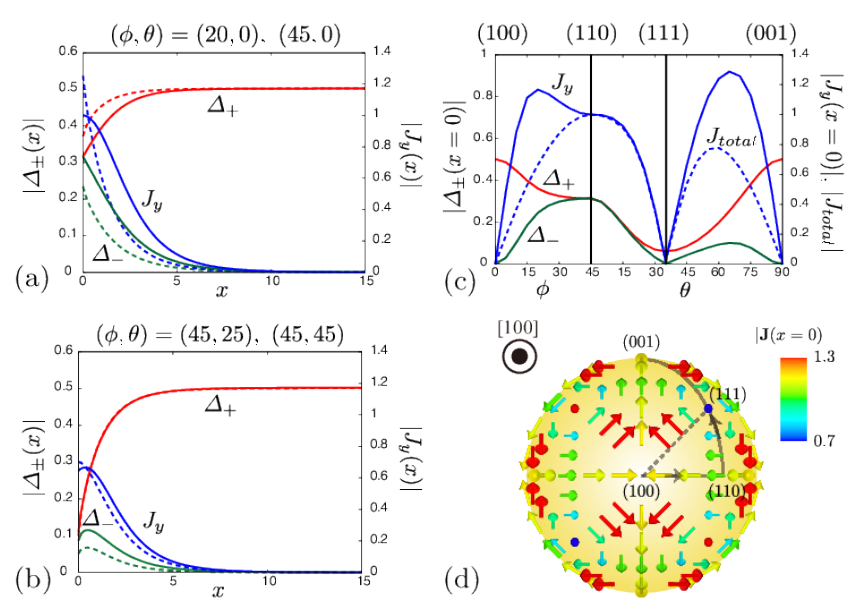

Fig. 1. (Color online) (a) Depth dependence of pair potential $\left|\Delta_{+}(x)\right|$, $\left|\Delta_{-}(x)\right|$, and spontaneous current $|J(x)|$ as a function of distance $x$ from the surface. Solid lines are for the surface orientation $(\phi, \theta)=\left(45^{\circ}, 0\right)$ and dashed lines are for $(\phi, \theta)=\left(20^{\circ}, 0\right) .\left|\Delta_{ \pm}\right|$is in the unit of $\pi k_{\mathrm{B}} T_{\mathrm{c}} \cdot|\mathbf{J}(x)|$ and $\left|\mathbf{J}_{\text {total }}\right|$ are, respectively, normalized by the values $|\mathbf{J}(x=0)|$ and $\left|\mathbf{J}_{\text {total }}\right|$ for $(\phi, \theta)=\left(45^{\circ}, 0\right)$. (b) Same as (a), but $(\phi, \theta)=\left(45^{\circ}, 25^{\circ}\right)$ for solid lines and $(\phi, \theta)=\left(45^{\circ}, 45^{\circ}\right)$ for dashed lines. (c) Surface orientation $(\phi, \theta)$ dependence of the surface state $\left|\Delta_{+}(x=0)\right|,\left|\Delta_{-}(x=0)\right|,|\mathbf{J}(x=0)|$ (solid lines), and $\left|\mathbf{J}_{\text {total }}\right|$ (dashed lines). The horizontal axis indicates that the surface orientation changes from $(1,0,0)$ to $(1,1,0)\left[\theta=0\right.$ and $\left.\phi=0 \rightarrow 45^{\circ}\right]$ and from $(1,1,0)$ to $(0,0,1)\left[\phi=45^{\circ}\right.$ and $\left.\theta=0 \rightarrow 90^{\circ}\right]$. (d) Schematic plot of spontaneous current at the surface of a large spherical sample. The line indicates the path of the horizontal axis in (c). The path $(1,1,1)-(0,0,1)$ is equivalent to the path $(1,1,1)-(1,0,0)$.

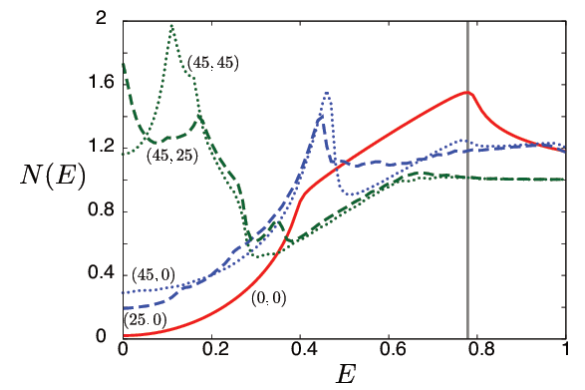

Fig. 2. (Color online) $\operatorname{LDOS} N(E, \mathbf{r})$ at surface $x=0$ with various surface orientations, $(\phi, \theta)=(0,0),\left(25^{\circ}, 0\right),\left(45^{\circ}, 0\right),\left(45^{\circ}, 25^{\circ}\right)$, and $\left(45^{\circ}, 45^{\circ}\right)$.

pressed, and the induced $\Delta_{-}$accompanies the $y$-component of the spontaneous current. These behaviors depend on the surface orientation. For the $(1,1,0)$ surface with $(\phi, \theta)=$ $\left(45^{\circ}, 0\right)$, since the $d_{x^{2}-y^{2}}$-wave component in the pairing function changes sign in the quasi-particle reflection at the surface, $\left|\Delta_{+}\right|=\left|\Delta_{-}\right|$at the surface so that the $d_{x^{2}-y^{2}}$-wave component vanishes there. On the other hand, for the $(1,0,0)$ surface with $(\phi, \theta)=(0,0), \Delta_{-}=0$ and $\mathbf{J}=0$ even near the surface, and the surface state is the same as the bulk state.

To confirm how the surface state depends on the surface orientation, in Fig. 1(c), we plot the surface values $\left|\Delta_{ \pm}(x=0)\right|$, $|\mathbf{J}(x=0)|$, and $\left|\mathbf{J}_{\text {total }}\right|$ along the trajectory $(1,0,0)-(1,1,0)$ $(1,1,1)-(0,0,1)$. Along $(1,0,0)-(1,1,0),\left|\Delta_{+}(x=0)\right|$ decreases and $\left|\Delta_{-}(x=0)\right|$ increases. $|\mathbf{J}(x=0)|$ increases and is maximum at $\phi=20^{\circ}$. However, the total current $\left|\mathbf{J}_{\text {total }}\right|$ monotonically increases until $\phi=45^{\circ}$. This is because the surface region in which spontaneous current appears becomes wider as $\phi$ approaches $45^{\circ}$, as seen in Fig. 1(a). Along $(1,1,0)-(1,1,1)$, $\left|\Delta_{ \pm}(x=0)\right|$ and current decrease. For the $(1,1,1)$ surface of the point node direction, $\Delta_{-}(x=0)=0$ and $J(x)$ vanishes. 


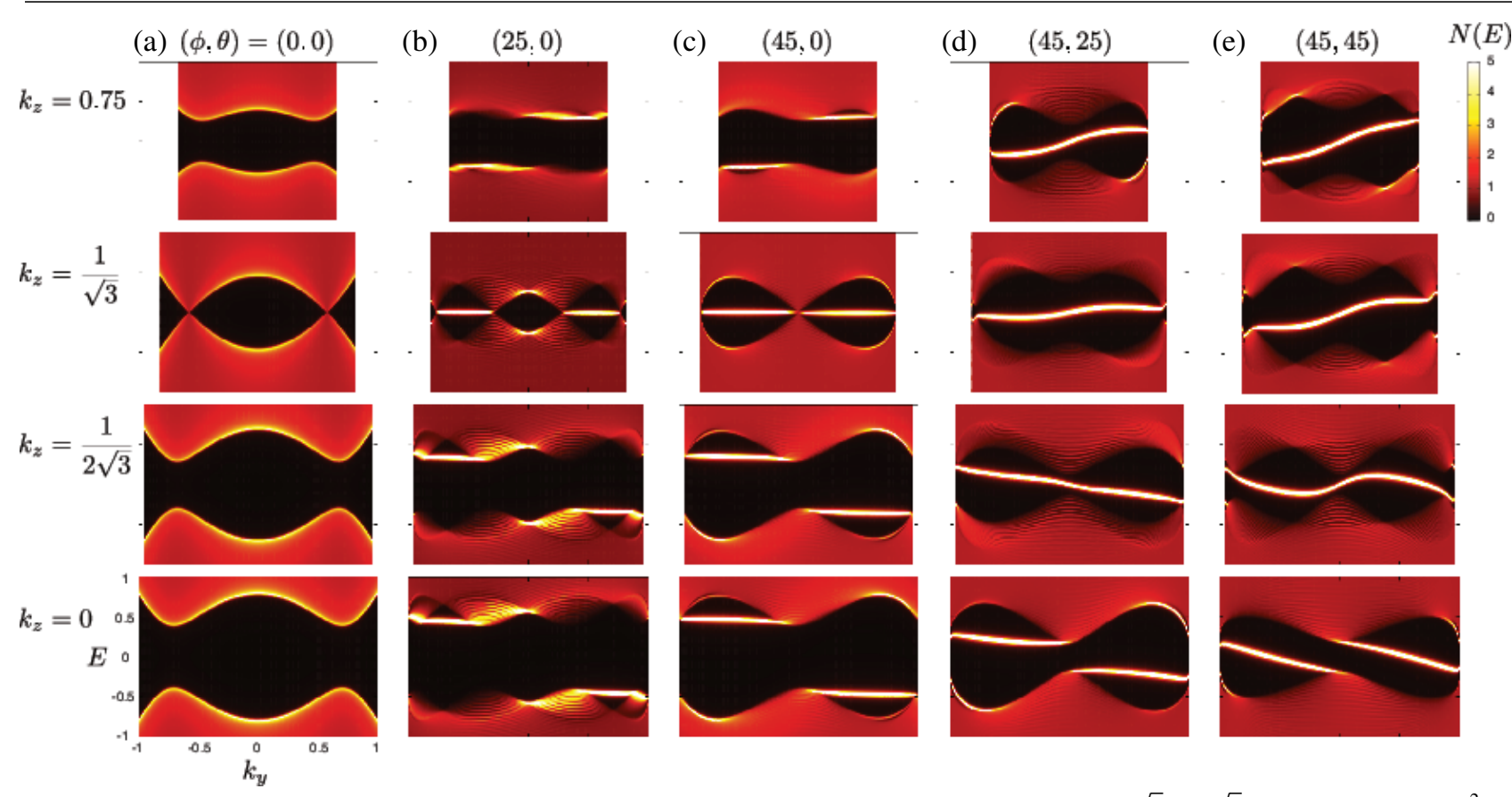

Fig. 3. (Color online) k-resolved $\operatorname{LDOS} N(E, \mathbf{k}, \mathbf{r})$ at surface $x=0$ as functions of $k_{y}$ and $E$ for $k_{z}=0,1 /(2 \sqrt{3}), 1 / \sqrt{3}$, and $0.75 . k_{x}=\left(1-k_{y}^{2}-k_{z}^{2}\right)^{1 / 2}$. Various surface orientations, $(\phi, \theta)=(0,0)(\mathrm{a}),\left(25^{\circ}, 0\right)(\mathrm{b}),\left(45^{\circ}, 0\right)(\mathrm{c}),\left(45^{\circ}, 25^{\circ}\right)(\mathrm{d})$, and $\left(45^{\circ}, 45^{\circ}\right)(\mathrm{e})$, are presented.

Along $(1,1,1)-(0,0,1)$, which is equivalent to $(1,1,1)-(1,0,0)$, $\left|\Delta_{+}(x=0)\right|$ increases monotonically. $\left|\Delta_{-}(x=0)\right|$ and current decrease after they increase.

In order to understand the flow of spontaneous current J, we consider the surface current in a large spherical superconductor of cyclic $d$-wave pairing. As in Fig. 1(d), at the point node direction $(1,1,1)$ and the equivalent points, $\mathbf{J}=0$. Around the node points $(1,1,1),(1,-1,-1),(-1,1,-1)$, and $(-1,-1,1), \mathbf{J}$ flows clockwise. Around other node points $(-1,-1,-1),(-1,1,1),(1,-1,1)$, and $(1,1,-1), \mathbf{J}$ flows counterclockwise. The direction of the flow is related to the angular momentum of the Cooper pairs via the phase winding of the spherical harmonic function, $Y_{2, m}$, on the Fermi sphere. The pairing function is expressed as $\phi_{+} \propto Y_{2,-2}-\mathrm{i} \sqrt{2} Y_{2,1}$ when $z \|(1,1,1)$ and as $\phi_{+} \propto Y_{2,2}+\sqrt{2} Y_{2,-1}$ when $z \|$ $(1,-1,1) \cdot{ }^{17}$ The spontaneous current at the surface is induced by the change in the angular momentum of Cooper pairs when they are reflected at the surface. By summing the spontaneous currents around neighboring point nodes, $\mathbf{J}$ is enhanced at $(1,1,0)$ and the equivalent positions. On the other hand, at $(1,0,0)$ and the equivalent positions, $\mathbf{J}$ is canceled to zero by summing neighboring spontaneous currents. These current distributions may be reflected in the spontaneous magnetic field distribution that is expected to be observed.

Next, we discuss electronic states at the surface. In Fig. 2, we present the $\operatorname{LDOS} N(E, x=0)$ for some cases of surface orientation. The LDOS is expected to be observed by tunneling spectroscopy at the surface. For the $(1,0,0)$ surface with $(\phi, \theta)=(0,0)$, surface states have the same electronic states as those in the bulk. There, $N(E) \propto E^{2}$ at low $E$ owing to point node excitations. The gap edge at $E \sim 0.8$ comes from the maximum of $\left|\Delta_{+} \phi_{+}(\mathbf{k})\right|$ at $\left(k_{a}, k_{b}, k_{c}\right) \propto(1,0,0)$. The hump at $E \sim 0.4$ corresponds to the saddle points of $\left|\Delta_{+} \phi_{+}(\mathbf{k})\right|$ at $\left(k_{a}, k_{b}, k_{c}\right) \propto(1,1,0)$.

With increasing $\phi$ from $(1,0,0)$ to $(1,1,0)$, low-energy surface bound states appear, including zero-energy states. The peak at $E \sim 0.8$ decays, and a new peak appears at $E \sim 0.5$. In the cases of surface orientations $(\phi, \theta)=\left(45^{\circ}, 25^{\circ}\right)$ and $\left(45^{\circ}, 45^{\circ}\right)$, the $\operatorname{LDOS} N(E, x=0)$ has high intensity at low energy, because both $\Delta_{+}(x=0)$ and $\Delta_{-}(x=0)$ are largely suppressed at the surface.

In order to understand the structures of the surface bound state and spontaneous current, we study the surface orientation dependence of the $\mathbf{k}$-resolved $\operatorname{LDOS} N(E, \mathbf{k}, \mathbf{r})$ at the surface $x=0$, as presented in Fig. 3, to examine the dispersion relation of surface bound states. For the $(1,0,0)$ surface with $(\phi, \theta)=(0,0)$, the surface state is the same as the bulk state. Thus, $N(E, \mathbf{k}, x=0)$ shows the gap structure of the pairing function $\left|\Delta_{+} \phi_{+}(\mathbf{k})\right|$. In the panel for $k_{z}=0$, we see the maximum of the gap $\left|\Delta_{+} \phi_{+}(\mathbf{k})\right|$ to be $E \sim 0.8$ at $k_{y}=0, \pm 1$, and the saddle point energy $E \sim 0.4$ at $k_{y}= \pm 1 / \sqrt{2}$. In the panel for $k_{z}=1 / \sqrt{3}$, zero energy states appear due to the point nodes at $k_{y}= \pm 1 / \sqrt{3}$.

With increasing $\phi$ from $(1,0,0)$ to $(1,1,0)$, in-gap states of the surface bound states appear. In the panel for $k_{z}=1 / \sqrt{3}$, flat dispersions of zero-energy modes exist at $0.28<\left|k_{y}\right|<$ 0.77 for the surface with $(\phi, \theta)=\left(25^{\circ}, 0\right)$. For the $(1,1,0)$ surface with $(\phi, \theta)=\left(45^{\circ}, 0\right)$, zero-energy modes extend to all $k_{y}$ at $k_{z}=1 / \sqrt{3}$. This is a property of the $d_{x^{2}-y^{2}}$-wave component, because the $d_{3 z^{2}-1}$-wave component vanishes at $k_{z}=1 / \sqrt{3}$. In Figs. 3(b) and 3(c), the flat dispersions are raised to finite energies at $k_{z} \neq 1 / \sqrt{3}$. When $k_{z}>1 / \sqrt{3}\left(k_{z}<\right.$ $1 / \sqrt{3})$, they appear at $E>0(E<0)$ for $k_{y}>0$ and at $E<0$ $(E>0)$ for $k_{y}<0$. Since a negative $E$ indicates an occupied state, the imbalance of negative $E$ states of $k_{y}>0$ and $k_{y}<0$ induces the spontaneous current. Since the contribution of the negative $E$ state of $k_{y}>0$ at $k_{z}<1 / \sqrt{3}$ is stronger than that of $k_{y}<0$ at $k_{z}>1 / \sqrt{3}$, this difference causes the spontaneous current to flow in the positive $y$ direction.

When the surface orientation changes from $(1,1,0)$ to $(0,0,1)$ upon increasing $\theta$ at $\phi=45^{\circ}$, the flat dispersions in Fig. 3(c) become dispersive, as shown in Figs. 3(d) and 


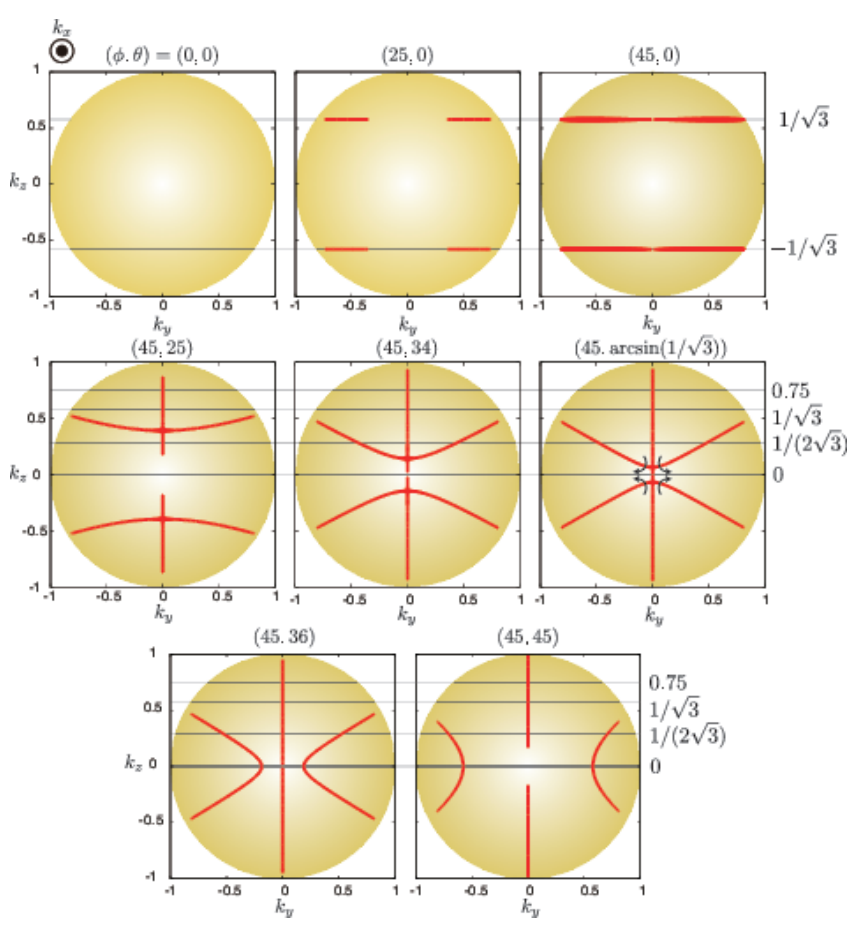

Fig. 4. (Color online) Bold lines indicate wave numbers $\left(k_{y}, k_{z}\right)$ of topological Fermi arcs satisfying the condition for zero-energy states, Eq. (9). $k_{x}=\left(1-k_{y}^{2}-k_{z}^{2}\right)^{1 / 2}$. Various surface orientation cases, $(\phi, \theta)=(0,0)$, $\left(25^{\circ}, 0\right),\left(45^{\circ}, 0\right),\left(45^{\circ}, 25^{\circ}\right),\left(45^{\circ}, 34^{\circ}\right),\left(45^{\circ}, \arcsin (1 / \sqrt{3})\right),\left(45^{\circ}, 36^{\circ}\right)$, and $\left(45^{\circ}, 45^{\circ}\right)$, are presented. The thin lines $k_{z}=0,1 /(2 \sqrt{3}), 1 / \sqrt{3}$, and 0.75 correspond to the horizontal axis in the panels in Fig. 3. The intersections of bold and thin lines indicate that the zero-energy states appear at $\left(k_{y}, k_{z}\right)$ in Fig. 3.

3(e). We see the reconnection of the dispersion curve between $\theta=0$ and $25^{\circ}$ in panels for $k_{z}=1 /(2 \sqrt{3})$ and 0.75 . The reconnection also occurs between $\theta=25^{\circ}$ and $45^{\circ}$ in the panel for $k_{z}=0$. Zero-energy states appear at $k_{y}=0$ in the upper three panels in both Figs. 3(d) and 3(e), indicating flat dispersion on part of the line $k_{y}=0$. In Fig. 3(e), we also see zero-energy states at $k_{y} \neq 0$ in the panels for $k_{z}=0$ and $k_{z}=1 /(2 \sqrt{3})$.

Lastly, we discuss the condition of $\mathbf{k}$ for zero-energy surface bound states to appear in the complex pairing function of cyclic $d$-wave superconductivity. We define the phase $\varphi_{\text {in }}$ of the pairing function before reflection at the surface as $\phi_{+}\left(-k_{x}, k_{y}, k_{z}\right)=\mid \phi_{+}\left(-k_{x}, k_{y}, k_{z}\right) \mathrm{e}^{\mathrm{i} \varphi_{\text {in }}}$ and the phase $\varphi_{\text {ref }}$ after reflection as $\phi_{+}\left(k_{x}, k_{y}, k_{z}\right)=\left|\phi_{+}\left(k_{x}, k_{y}, k_{z}\right)\right| \mathrm{e}^{\mathrm{i} \varphi_{\text {ref }}}$. As the condition under which the zero-energy surface bound states in Fig. 3 are well explained, we find the relation

$$
\mathrm{e}^{\mathrm{i} \varphi_{\text {in }}}=-\mathrm{e}^{\mathrm{i} \varphi_{\text {ref }}},
$$

i.e., the $\pi$-phase shift $\varphi_{\text {ref }}-\varphi_{\text {in }}=\pi(\bmod 2 \pi) \cdot{ }^{19}$ Equation (9) can be applied even when $\left|\phi_{+}\left(-k_{x}, k_{y}, k_{z}\right)\right| \neq\left|\phi_{+}\left(k_{x}, k_{y}, k_{z}\right)\right|$.

Wave numbers $\mathbf{k}$ satisfying Eq. (9) are presented in Fig. 4. These topological Fermi arcs are terminated at the point node directions. ${ }^{3}$ For the $(1,0,0)$-surface with $(\phi, \theta)=(0,0)$, there are no zero-energy surface states. For the $(1,1,0)$-surface with $(\phi, \theta)=\left(45^{\circ}, 0\right)$, all $k_{y}$ values satisfy Eq. (9) on the lines $k_{y}= \pm 1 / \sqrt{3}$. When changing $\phi$ from $(1,0,0)$ to $(1,1,0)$, the region of $k_{y}$ in which zero-energy surface bound states appear increases. These reproduce the behavior of the zero-energy flat dispersion in the panels for $k_{z}=1 / \sqrt{3}$ in Figs. 3(a)-3(c). When changing $\theta$ from $(1,1,0)$ to $(0,0,1)$, the lines of zero energy at $k_{z}=1 / \sqrt{3}$ are shifted to smaller $\left|k_{z}\right|$ and become diagonal lines. Zero-energy states also appear on the vertical line $k_{y}=0$. The endpoints of the zero-energy states on the vertical line are the point node directions. When $\theta$ approaches $\arcsin (1 / \sqrt{3}) \sim 35^{\circ}$ of the $(1,1,1)$-surface direction, all vertical and diagonal lines pass through the center $\left(k_{y}, k_{z}\right)=(0,0)$. The reconnection of these lines occurs at this point-node wave number, as shown in Fig. 4. From the panel for $(\phi, \theta)=\left(45^{\circ}, 25^{\circ}\right)$, we find that zero-energy states appear at $k_{y}=0$ in the plots along the lines $k_{z}=1 /(2 \sqrt{3}), 1 / \sqrt{3}$, and 0.75 . From the panel for $(\phi, \theta)=\left(45^{\circ}, 45^{\circ}\right)$, zero-energy states are seen to also appear at $k_{y} \neq 0$ in the plots along $k_{z}=0$ and $1 /(2 \sqrt{3})$. These well explain the wave numbers of the zero-energy states in Figs. 3(d) and 3(e).

In summary, we studied the surface orientation dependence of the surface states in the cyclic $d$-wave superconductor, as an example of the BTRS state in cubic lattice symmetric superconductivity. There, spontaneous currents flow around each point node direction if we prepare spherical samples. We also identified the condition under which topological Fermi arcs of zero-energy surface bound states appear in cyclic $d$ wave superconductors. These are useful results for studying new types of BTRS superconductors other than those with chiral $p$-wave pairing.

We thank T. Mizushima and T. Kawakami for fruitful discussions. This work was supported by KAKENHI Grants No. 21340103 and No. 24840048.

1) M. Stone and R. Roy: Phys. Rev. B 69 (2004) 184511.

2) Y. Tsutsumi, M. Ichioka, and K. Machida: Phys. Rev. B 83 (2011) 094510; Y. Tsutsumi, T. Mizushima, M. Ichioka, and K. Machida: J. Phys. Soc. Jpn. 79 (2010) 113601.

3) M. A. Silaev and G. E. Volovik: Phys. Rev. B 86 (2012) 214511; G. E. Volovik: arXiv:1110.4469.

4) G. M. Luke, A. Keren, L. P. Le, W. D. Wu, Y. J. Uemura, D. A. Bonn, L. Taillefer, and J. D. Garrett: Phys. Rev. Lett. 71 (1993) 1466.

5) A. P. Mackenzie and Y. Maeno: Rev. Mod. Phys. 75 (2003) 657.

6) Y. Aoki, A. Tsuchiya, T. Kanayama, S. R. Saha, H. Sugawara, H. Sato, W. Higemoto, A. Koda, K. Ohishi, K. Nishiyama, and R. Kadono: Phys. Rev. Lett. 91 (2003) 067003.

7) Y. Aoki, T. Tayama, T. Sakakibara, K. Kuwahara, K. Iwasa, M. Kohgi, W. Higemoto, D. E. MacLaughlin, H. Sugawara, and H. Sato: J. Phys. Soc. Jpn. 76 (2007) 051006.

8) A. D. Hillier, J. Quintanilla, and R. Cywinski: Phys. Rev. Lett. 102 (2009) 117007.

9) A. Maisuradze, W. Schnelle, R. Khasanov, R. Gumeniuk, M. Nicklas, H. Rosner, A. Leithe-Jasper, Yu. Grin, A. Amato, and P. Thalmeier: Phys. Rev. B 82 (2010) 024524.

10) A. D. Hillier, J. Quintanilla, B. Mazidian, J. F. Annett, and R. Cywinski: Phys. Rev. Lett. 109 (2012) 097001.

11) G. E. Volovik and L. P. Gor'kov: Pis'ma Zh. Eksp. Teor. Fiz. 39 (1984) 550 [JETP Lett. 39 (1984) 674]; G. E. Volovik and L. P. Gor'kov: Zh. Eksp. Teor. Fiz. 88 (1985) 1412 [Sov. Phys. JETP 61 (1985) 843].

12) M. Sigrist and K. Ueda: Rev. Mod. Phys. 63 (1991) 239.

13) M. Ozaki, K. Machida, and T. Ohmi: Prog. Theor. Phys. 74 (1985) 221

14) G. W. Semenoff and F. Zhou: Phys. Rev. Lett. 98 (2007) 100401.

15) H. Mäkelä: J. Phys. A 39 (2006) 7423.

16) M. Kobayashi, Y. Kawaguchi, M. Nitta, and M. Ueda: Phys. Rev. Lett. 103 (2009) 115301.

17) H. M. Adachi, Y. Tsutsumi, J. A. M. Huhtamäki, and K. Machida: J. Phys. Soc. Jpn. 78 (2009) 113301; H. M. Adachi, Y. Tsutsumi, and K. Machida: J. Phys. Soc. Jpn. 79 (2010) 044301.

18) Y. Tanaka and S. Kashiwaya: Phys. Rev. Lett. 74 (1995) 3451.

19) S. Kashiwaya and Y. Tanaka: Rep. Prog. Phys. 63 (2000) 1641.

20) M. Sato, Y. Tanaka, K. Yada, and T. Yokoyama: Phys. Rev. B 83 (2011) 224511. 